\title{
Cathelicidin LL37: Defense Roles in the Early Stages of Mycobacterium Tuberculosis Infection
}

\author{
Kai Zhang ${ }^{1,2}$, Jie Wang², Xiangmei Zhou*2 and Lihua $\mathrm{Xu}^{* 1}$ \\ ${ }^{1}$ School of Agriculture, Ningxia University, China \\ ${ }^{2}$ National Animal Transmissible Spongiform Encephalopathy Laboratory, China Agricultural University, China
}

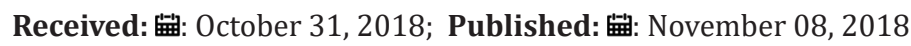

*Corresponding author: Xiangmei Zhou, National Animal Transmissible Spongiform Encephalopathy Laboratory, College of Veterinary Medicine, China Agricultural University, Beijing, China

Lihua Xu, School of Agriculture, Ningxia University, Yinchuan, Ningxia, China

\begin{abstract}
Mycobacterium tuberculosis (MTB) has developed into a very successful pathogen through a long history of co-evolution with humans, which can manipulate the body's immune system for its persistent survival. The susceptibility of this disease depends to a large extent on the relationship between the host immune system and the virulence of MTB. Cathelicidin LL37 plays a vital role in innate immune response and can resist the invasion of MTB. This article focuses on the role of cathelicidin LL37 in against MTB infection in vivo.
\end{abstract}

Abbreviations: MTB: Mycobacterium Tuberculosis; HDPs: Host Defense Peptides; BCG: Bacillus Calmette Guerin; EGFR: Epidermal Growth Factor Receptor; NETs: Neutrophil Extracellular Traps; FPRL1: Formyl Peptide Receptor-Like 1

\section{Introduction}

Tuberculosis (TB), caused by Mycobacterium tuberculosis (MTB), is the most deadly infectious diseases. In 2017, about 10 million people worldwide infected with TB and 1.6 million deaths due to TB. With the emergence of drug-resistant tuberculosis and multidrug resistant tuberculosis (MDR-TB), it is more difficult to prevent and control TB [1]. As an intracellular pathogen, MTB first triggers the innate immune response during infection and is phagocytosed by macrophages and dendritic cells, and is then degraded into immunogenic polypeptides and presented on $\mathrm{T}$ lymphocytes by major histocompatibility complexes to trigger adaptive immunity [2]. However, MTB has developed a wide type of strategies to evade the bactericidal activities of these cells, enabling it to successfully establish a long-lived niche in macrophages [3]. Several molecules of the innate immune system are involved in the process of interaction between the host and MTB, including host defense peptides (HDPs), such as cathelicidin and defensins.

Cathelicidin are a family of small cationic peptides that have diverse roles in the immune system. LL-37 is the only known human cathelicidin, primarily produced by the active domains of macrophages, monocytes, neutrophils, epithelial cells [4]. In addition to its antibacterial activity, LL-37 also has a variety of biological activities, including regulation of inflammation, antitoxin, anti-tumor, wound healing and other functions [5]. Current studies have found that LL37 kills MTB mainly in these ways:
a. Direct killing
b. Regulation of inflammation
c. Promotion of macrophage autophagy

\section{Antibacterial Activity of LL37}

LL37 has a broad spectrum of antimicrobial activity and can quickly and effectively kill G+ bacteria, G- bacteria, fungi and some enveloped viruses. Several studies revealed that LL-37 uses a toroidal pore carpet-like mechanism commonly used by other cationic antimicrobial peptides, in which the peptides insert in the outer membrane of the pathogen in a perpendicular manner, forming pores and causing severe leakage that leading to the death of the pathogen [6]. Torresjuarez [7] reported that exogenous LL37 suppresses the survival of MTB strain H37Rv in monocyte-derived macrophages. Lucille [8] confirmed that C57BL/6 mice are more resistant to infection compared with $\mathrm{BALB} / \mathrm{c}$ mice within three days after intranasal inoculation with Bacillus Calmette-Guerin (BCG), which was associated with high CRAMP (a similar substance of LL37 in mice) expression in the lungs. In addition, the LL37/DNA complex produced by the Neutrophil Extracellular Traps (NETs) enters human peripheral blood mononuclear macrophages via the P2RX7 receptor and clathrin-mediated endocytosis, followed by Bactericidal action of BCG by lysosomes [9]. 


\section{LL37 Regulates Inflammatory Response}

The body's immune system resists MTB through inflammatory cells and inflammatory factors during MTB infection. LL37 plays a key role in the body's proinflammatory and anti-inflammatory mechanisms. Several studies have found that the concentration of LL37 is significantly increased in the site of inflammation in the body, such as skin, lungs, and oral cavity [10]. LL37 can drive biological responses through epidermal growth factor receptor (EGFR), formyl peptide receptor-like 1 (FPRL1), TLR4 and TLR9, induce the production of chemokines and pro-inflammatory cytokines, recruit and activate inflammatory cells, including granulocytes, monocytes, macrophages, eosinophils and mast cells. In addition, LL37 promotes mast cell degranulation and enhances NK cell function [11]. Other studies have found that LL37 can inhibit the apoptosis of neutrophils, prolong their life span, and promote the formation of NETs [12]. In addition, LL-37 interacts with cell membranes, affects cell surface receptors, and carries DNA or double-stranded RNA into cells, activating a variety of intracellular signaling pathways [13].

\section{LL37 Promotes Autophagy In Macrophages}

Cellular autophagy is a programmed homeostatic cellular process, which plays a key role to maintain the balance between protein synthesis and degradation in eukaryotic cells. Autophagy is a lysosome-dependent degradation pathway that widely exists in cells. It not only can degrade damaged proteins and organelles in cells, but also involves the removal of harmful components in the cell, which is conducive to the recycling of substances and resists the invasion of harmful substances. The most prominent role of autophagy in relation to immunity is to kill intracellular pathogens such as MTB [14]. During H37RV infection, LL37 promotes macrophage autophagy and kills H37RV by activating P2RX7 receptor and intracellular free $\mathrm{Ca} 2+$ pathway, AMPK signaling pathway and PI3K signaling pathway [15].

\section{Summary}

Thus, as part of the host's innate immunity, LL37 plays an important role in the fight against MTB infection. However, MTB can inhibit the expression of cathelicidin LL37 by subverting the cAMP signaling pathway, thereby evading the innate immune response, which is detrimental to the body's resistance to MTB infection [16]. Fortunately, the current study found that exogenous addition of VD3, Butyrate, astragalus polysaccharides, resveratrol and other substances can promote the expression of LL37 in vivo. This provides new ideas for researchers to prevent and treat TB.

\section{References}

1. (2018) World Health Organization, Global tuberculosis report.
2. Bose Dasgupta S, Pieters J (2018) Macrophage-microbe interaction: lessons learned from the pathogen Mycobacterium tuberculosis. Semin Immunopathol 40(6): 577-591.

3. Ehrt S, Schnappinger D, Rhee KY (2018) Metabolic principles of persistence and pathogenicity in Mycobacterium tuberculosis. Nature Reviews Microbiology 16(8): 496-507.

4. Talukder P, Satho T, Irie K, Sharmin T, Hamady D, et al. (2011) Trace metal zinc stimulates secretion of antimicrobial peptide LL-37 from caco-2 cells through erk and p38 map kinase. International Immunopharmacology 11(1): 141-144.

5. Zhang LJ, Sen GL, Ward NL, Johnston A, Chun K, et al. (2016) Antimicrobial peptide LL-37 and mavs signaling drive interferon- $\beta$ production by epidermal keratinocytes during skin injury. Immunity 45(1): 119-130.

6. Vandamme D, Landuyt B, Luyten W, Schoofs L (2012) A comprehensive summary of LL-37, the factotum human cathelicidin peptide. Cellular Immunology 280(1): 22-35.

7. Torresjuarez F, Cardenasvargas A, Montoyarosales A, Gonzálezcuriel I, Garciahernandez MH, et al. (2015) LL-37 immunomodulatory activity during mycobacterium tuberculosis infection in macrophages. Infection \& Immunity 83(12): 4495.

8. Adam L, López González M, Björk A, Pålsson S, Poux C, et al. (2018) Early Resistance of Non-virulent Mycobacterial Infection in C57BL/6 Mice Is Associated with Rapid Up-Regulation of Antimicrobial Cathelicidin Camp. Front. Immunol 9: 1939.

9. Stephan A, Batinica M, Steiger J, Hartmann P, Zaucke F, et al. (2016) LL37: DNA complexes provide antimicrobial activity against intracellular bacteria in human macrophages. Immunology 148(4): 420-432.

10. Brown KL, Poon GF, Birkenhead D, Pena OM, Falsafi R, et al. (2011) Host defense peptide LL37 selectively reduces proinflammatory macrophage responses. Journal of Immunology 186(9): 5497-5505.

11. Agier J, Różalska S, Wiktorska M, Żelechowska P, Pastwińska J, et al. (2018) The RLR/NLR expression and pro-inflammatory activity of tissue mast cells are regulated by cathelicidin LL-37 and defensin HBD2. Scientific Reports 8: 11750.

12. Nagaoka I, Tamura H, Hirata M (2006) An Antimicrobial Cathelicidin Peptide, Human CAP18/LL-37, Suppresses Neutrophil Apoptosis via the Activation of Formyl-Peptide Receptor-Like 1 and P2X7. J Immunol. 176(5): 3044-3052.

13. Merkle M, Pircher J, Mannell H, Krötz F, Blüm P, et al. (2015) LL37 inhibits the inflammatory endothelial response induced by viral or endogenous DNA. Journal of Autoimmunity 65: 19-29.

14. Floresvaldez MA, Seguracerda CA, Gaonabernal J (2018) Modulation of autophagy as a strategy for development of new vaccine candidates against tuberculosis. Molecular Immunology. 97: 16-19.

15. Rekha RS, Rao MS, Wan M, Raqib R, Bergman P, et al. (2015) Phenylbutyrate induces LL-37 dependent autophagy and intracellular killing of mycobacterium tuberculosis in human macrophages. Autophagy 11(9): 1688-1699.

16. Gupta S, Winglee K, Gallo R, Bishai WR (2017) Bacterial subversion of camp signaling inhibits cathelicidin expression, which is required for innate resistance to mycobacterium tuberculosis. Journal of Pathology 242(1): 52-61. 
ISSN: 2574-1241

DOI: $10.26717 / B J S T R .2018 .10 .002013$

Xiangmei Zhou. Biomed J Sci \& Tech Res

CC (i) This work is licensed under Creative

Submission Link: https://biomedres.us/submit-manuscript.php

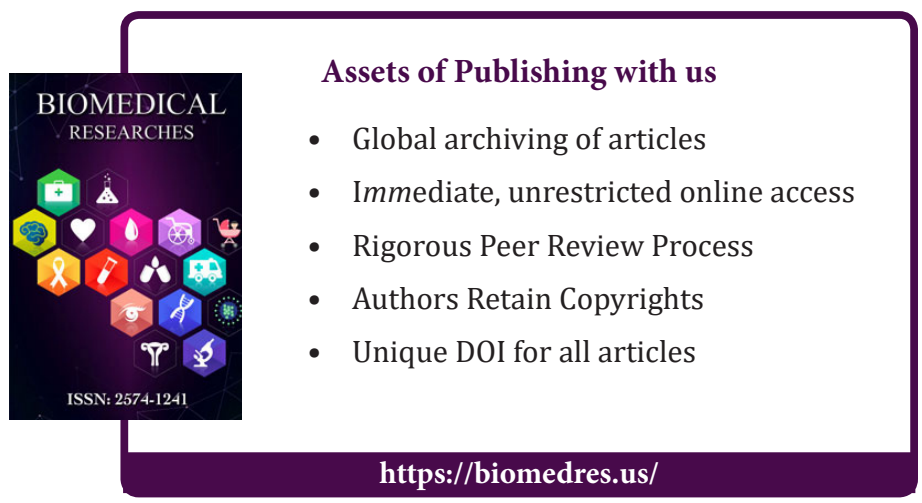

